\title{
Striatal Neuron Differentiation from Neurosphere-Expanded Progenitors Depends on Gsh2 Expression
}

\author{
Josephine B. Jensen, Anders Björklund, and Malin Parmar \\ Wallenberg Neuroscience Center, Section of Neurobiology, and Lund Strategic Research Center for Stem Cell Biology and Cell Therapy, Lund University, \\ SE-221 84 Lund, Sweden
}

\begin{abstract}
Neural stem and progenitor cells from the embryonic forebrain can be expanded under growth factor stimulation in vitro, either as free-floating aggregates called neurospheres or as attached monolayer cultures. We have previously shown that despite the maintenance of important regulatory genes such as Gsh2, in vitro expansion of cells from the lateral ganglion eminence (LGE) restricts their differentiation potential. Specifically, their ability to differentiate into striatal projection neurons is compromised. It is not clear whether this restriction is caused by loss of progenitors with the ability to generate striatal projection neurons or whether the restricted differentiation potential is caused by factors lacking during in vitro differentiation. To address this, we have set up an in vitro system, in which expanded LGE-derived cells are differentiated in coculture with primary cells isolated from different regions of the embryonic brain. We provide evidence that the primary cells supply the expanded cells with contact-mediated region-specific developmental cues. Neurosphereexpanded LGE progenitors can, when presented with these cues, differentiate into neurons with characteristics of striatal projection neurons. Furthermore, we show that the ability of the expanded LGE cells to respond to the developmental cues presented by the primary cells depends on the maintained expression of Gsh2 in the expanded cells.
\end{abstract}

Key words: LGE; Er81; ISL1; DARPP-32; mouse; region specific; coculture

\section{Introduction}

Striatal projection neurons and olfactory bulb interneurons are derived from the same embryonic structure, the lateral ganglionic eminence (LGE) (Deacon et al., 1994; Olsson et al., 1995, 1997, 1998; Wichterle et al., 1999, 2001). The dopamine and cAMPregulated phosphoprotein (DARPP-32)-expressing striatal projection neurons are generated almost exclusively during the embryonic period (Bayer and Altman, 1995) from a progenitor population in the LGE subventricular zone marked by the expression of the LIM homeodomain protein ISL1 (Thor et al., 1991; Toresson et al., 2000; Toresson and Campbell, 2001; Wang and Liu, 2001; Yun et al., 2003). Genetic evidence suggests that both this striatal progenitor population and the olfactory bulb progenitor population are derived from the GSH2-expressing cells of the LGE ventricular zone (Corbin et al., 2000; Toresson et al., 2000; Toresson and Campbell, 2001; Yun et al., 2001, 2003).

When neural precursor cells are isolated from embryonic or adult brain and expanded in vitro under mitogen stimulation, the

\footnotetext{
Received Jan. 22, 2004; revised June 22, 2004; accepted June 23, 2004.

This work was supported by grants from the Swedish Research Council (04X-3874 and 99SN-14480). Lund Stem Cell Center is supported by a Center of Excellence Grant from the Swedish Foundation for Strategic Research. We thank AnnaKarin Olden and Elsy Ling for technical assistance; Dr. M. Okabe for the $\beta$-actin GFP mice; Dr. S. Potter for the Gsh2-I- mice; Dr. C. Skogh for the ROSA cells; Drs. K Campbell, H. Edlund, T. Edlund, P. Greengard, T. M. Jessell, and S. Morton for their gift of antibodies; and Lee Rubin (Curis, Inc.) for supplying the SHH protein. We also thank Drs. K. Campbell and C. Skogh for helpful discussions and comments on this manuscript. The vectors used in this study were produced by Lund University Vector Unit (RVEC).

Correspondence should be addressed to M. Parmar, Wallenberg Neuroscience Center, Physiological Sciences, BMC A11, Lund 221 84, Sweden. E-mail: Malin.Parmar@mphy.lu.se.

D01:10.1523/JNEUROSCI.1331-04.2004

Copyright $\odot 2004$ Society for Neuroscience $\quad$ 0270-6474/04/246958-10\$15.00/0
}

expanded cells maintain many aspects of their regional identity (Zappone et al., 2000; Yamamoto et al., 2001; Hitoshi et al., 2002; Parmar et al., 2002). However, we have previously shown that the differentiation potential of growth factor-expanded LGE cells are compromised: the expanded LGE cells generate neurons with characteristics of olfactory bulb interneurons but not striatal projection neurons under standard in vitro differentiation conditions (Skogh et al., 2001, 2003; Parmar et al., 2002). This might suggest that the progenitors of striatal projection neurons are not expandable in culture. Alternatively, the cultures may contain the striatal progenitor population, but these expanded progenitors need to be presented with the correct developmental cues, which are not present during in vitro differentiation, to mature into striatal neurons in culture.

In primary culture from the LGE, in which the striatal ventricular and subventricular zone cells are differentiated in the absence of growth factors, both ISL1 and DARPP-32 expression is maintained (Ivkovic and Ehrlich, 1999; Toresson et al., 1999; Skogh et al., 2003). This suggests that the endogenous signals necessary for striatal neuron differentiation may be present in the primary LGE cultures. To explore this possibility and to investigate whether the developmental cues present in the primary cultures can direct striatal projection neuron differentiation from expanded LGE cells, we differentiated expanded cells in coculture with primary cells.

We show that in these cocultures, primary LGE cells are able to guide the differentiation of neurosphere-expanded LGE cells toward a striatal projection neuron-like fate. The signal or signals provided by the primary cells are region specific and require direct cell-to-cell contact. We also provide evidence that the ability 
of the expanded cells to respond to the developmental cues in the cocultures critically depends on Gsh2 expression. We therefore suggest that the principal striatal progenitor population present in long-term expanded neurosphere cultures is the Gsh2expressing cell population.

\section{Materials and Methods}

Dissection and preparation of single-cell suspension

All animal-related procedures were conducted in accordance with local ethical guidelines and approved animal care protocols. LGE, medial ganglionic eminence (MGE), and cortex (CTX) were dissected from embryonic day 13.5 (E13.5) mouse embryos, and ventral mesencephalon (VM) was dissected from E12.5 embryos. The tissue pieces were incubated in basic medium for $15 \mathrm{~min}$ at $37^{\circ} \mathrm{C}$, followed by mechanical dissociation. Live cells were counted by trypan blue dye exclusion method before plating as either primary or expansion cultures.

\section{Expansion cultures}

Neurospheres. LGE neurosphere cultures were generated from timed pregnant wild-type mice or from mice expressing enhanced green fluorescent protein (EGFP) under the cytomegalovirus (CMV)- $\beta$-actin promoter (courtesy of Dr. M. Okabe). Isolated cells were plated at a concentration of 100,000 cells/ml in basic medium, DMEM-F-12 supplemented with a defined hormone and salt mixture (Reynolds et al., 1992), containing $10 \mathrm{ng} / \mathrm{ml}$ basic fibroblast growth factor and $20 \mathrm{ng} / \mathrm{ml}$ epidermal growth factor (EGF). Cells were passaged once a week by mechanical dissociation and replated at a density of 50,000 cells/ml. No apparent differences in growth rate or appearance between the wild-type and transgene-expressing neurosphere cultures were observed.

Gsh2-/- neurospheres. Cultures from individual LGEs were started in 24-well plates containing the same neurosphere-expansion medium as described above. At the time of dissection, a tissue piece from each embryo was also recovered and used for genotyping of the embryos by PCR as previously described (Szucsik et al., 1997). The cultures were coded, and all experiments were performed blind.

Monolayer cultures. Monolayer expansion cultures were generated from ROSA26 mice (The Jackson Laboratory, Bar Harbor, ME), as previously described (Skogh et al., 2001). In brief, the cultures were expanded in basic medium supplemented with $10 \%$ serum and $20 \mathrm{ng} / \mathrm{ml}$ EGF. The cells were passaged 1:3 by trypzination when confluent.

Retroviral infection. Wild-type neurospheres at passage 5 and Gsh2+/- and Gsh2-1- neurospheres at passage 7 were mechanically dissociated into single cells. Six hours after passage, the cells were labeled using a recombinant VSV-G pseudotyped retroviral vector expressing EGFP under the CMMP LTR modified from (Ory et al., 1996). Cells were transduced by adding the virus (multiplicity of infection of 1) to the expansion medium. Five to six days after passage and infection, the spheres were used for the coculture experiments.

\section{Cocultures with primary cells}

Primary cell cultures were generated by plating single-cell suspensions obtained from LGE, MGE, CTX, or VM as described above. The cells were plated at a density of 120,000 cells $/ \mathrm{cm}^{2}(200,000$ cells/well $)$ in polyL-lysine (PLL)-coated 4-well chamber slides in basic medium supplemented with $1 \%$ serum (differentiation medium). Twenty-four hours after plating, when the primary cells had attached to the bottom of the wells, the expanded GFP-expressing (neurospheres) or LacZ-expressing (monolayer) LGE cells were added to the unlabeled primary cells. All coculturing of expanded cells was done on passage 5-8 cultures, and 100,000 expanded cells or equivalent were added per well. The cells were grown in coculture for 3,7 , or $10 \mathrm{~d}$ before fixation.

For quantification, the expanded cells were detected by GFP expression. Thirty predetermined evenly spread stops was made per well. The total number of GFP-expressing cells, the number of GFP cells with neuronal morphology, and the number of GFP-expressing cells with neuronal morphology that coexpressed ISL1 or Er81 was counted at each stop. The percentage of GFP-expressing cells with neuronal morphology of the total number of GFP-expressing cells and the percentage of these that coexpressed ISL1 or Er81 was determined for each well. Cells in at least five wells from two or three separate coculture experiments were quantified.

In vitro differentiation with brain-derived neurotrophic factor, retinoic acid, sonic hedgehog, and neurotrophin-3

LGE-derived neurospheres were plated on PLL-coated 4-well chamber slides or in coculture with LGE primary cells in standard differentiation medium. Brain-derived neurotrophic factor (BDNF) $(100 \mathrm{ng} / \mathrm{ml})$, retinoic acid (RA) (100 nM), sonic hedgehog (SHH) $(2 \mu \mathrm{g} / \mathrm{ml}, 1 \mu \mathrm{g} / \mathrm{ml}, 0.5$ $\mu \mathrm{g} / \mathrm{ml}$, and $0.1 \mu \mathrm{g} / \mathrm{ml}$; supplied by Lee Rubin, Curis, Inc.), or neurotrophin-3 (NT-3) $(50 \mathrm{ng} / \mathrm{ml})$ were added to the differentiation medium alone, or in combination as stated. RA was replenished every 24 $\mathrm{hr}$, and BDNF, SHH, and NT-3 were replenished every $2-3 \mathrm{~d}$. Cells were fixed after $7 \mathrm{~d}$ of differentiation.

\section{Conditioned medium assay}

Primary cells from LGE and passage 5-6 LGE neurospheres were differentiated in parallel in separate wells, but under the same conditions as described for the cocultures. Every $48 \mathrm{hr}, 50 \%$ of the medium of the differentiating neurospheres was removed and replaced with conditioned medium (CM) from the primary cells. The CM was filtered using low-binding durapore polyvinylidene difluoride membrane filter, pore size $5.0 \mu \mathrm{m}$ (Millipore, Bedford, MA), to remove any contaminating cells. Equal volume fresh medium was then added to the primary cells. As positive control, $50 \%$ of the medium in a standard coculture assay was renewed every $48 \mathrm{hr}$.

\section{Two-chamber, shared medium assay}

Primary cells from LGE were plated at a density of 200,000 cells/well in poly-D-lysine-PLL-coated 8-well chamber slides in differentiation medium. Twenty-four hours after plating, $\sim 50,000$ neurosphere-expanded LGE cells were added to an adjacent chamber separated by a low wall. Two hours later, when the neurospheres had attached, the medium was raised to a level, where it was shared between the two adjacent chambers. Cells were fixed after $7 \mathrm{~d}$ of differentiation.

\section{Immunocytochemistry}

All cultures were fixed in ice-cold 4\% PFA (plus glutaraldehyde for GABA stainings) for $15 \mathrm{~min}$ at room temperature. After rinsing with PBS, cultures were preincubated in 5\% normal serum and $0.25 \%$ Triton X-100 for $1 \mathrm{hr}$ at room temperature. Primary antibodies used were: rabbit anti$\beta$-III-tubulin (1:2000; Covance), mouse anti- $\beta$-III-tubulin (1:250; Sigma, St. Louis, MO), mouse anti-CNPase (1:100; Sigma), rabbit antiGFAP (1:1000; Dako, Carpinteria, CA), mouse anti-DARPP-32 (1:20,000; provided by P. Greengard), rabbit anti-GABA (1:5000; Incstar, Stillwater, MN), mouse anti-ISL1/2 (1:400; provided by H. and T. Edlund), rabbit anti-Er81 (1:2000; provided by T. M. Jessell and S. Morton), rabbit anti-TH (1:1000; Pelfreeze), mouse anti- $\beta$-galactosidase (1:500; Promega, Madison, WI), rabbit anti- $\beta$-galactosidase (1:500; 5 Prime $\rightarrow 3$ Prime, Inc., Boulder, CO), and chicken anti-GFP (1:5000; Chemicon, Temecula, CA).

Incubations in primary antisera were performed overnight at room temperature. Detection with secondary antibodies was performed using standard protocols. Both fluorescent (1:400) and biotin-conjugated (1: 200) antibodies were diluted in the preincubation solution and incubated for 1-2 hr at room temperature. The biotinylated antibodies were detected using the $\mathrm{ABC}$ method (Vector Laboratories, Burlingame, $\mathrm{CA}$ ) with diaminobenzidine (DAB) as the final chromogen.

\section{Statistical analysis}

The results from each experiment were combined, and all results are reported as value \pm SEM. Significance was tested for by one-way ANOVA, followed by Bonferroni post hoc test with $p<0.05$.

\section{Results}

Differentiating expanded cells in coculture with primary LGE cells do not effect the differentiation of neurons and oligodendrocytes

Neurospheres were generated from the E13.5 LGE of wild-type NMRI or transgenic mice expressing EGFP under the control of 
the chicken $\beta$-actin promoter and CMV enhancer (Okabe et al., 1997; Hadjantonakis et al., 1998). Before coculture, the neurospheres derived from wild-type mice were labeled with EGFP by retroviral infection, hence, in both cases cells originating from neurospheres could be distinguished from the primary cells by the expression of GFP.

When the neurospheres derived from the transgenic mouse were differentiated in coculture with primary cells, GFP expression was detected primarily in cells with astrocyte-like morphologies (Fig. $1 \mathrm{~A}$, green, arrow), but also in a substantial number of cells with neuron-like (Fig. $1 \mathrm{~A}$, green, arrowhead) morphologies. The majority of cells with neuron-like morphologies were found to coexpress $\beta$-III-tubulin (Fig. $1 A$, red). In virally infected neurosphere cultures, $\sim 25 \%$ of the total cells and $50 \%$ of the $\beta$-III-tubulin-positive neurons expressed GFP (Fig. $1 B$ ).

In our cocultures, 100,000 neurosphere cells are differentiated on top of 200,000 primary cells. After differentiation, the majority of the cells expressed GFAP and had the morphology of astrocytes (data not shown). Differentiating the expanded cells in coculture with primary cells did increase the numbers of $\beta$-III-tubulin-positive neurons formed from expanded cells from $\sim 4$ to $\sim 8 \%$ (Fig. 1C). Because neurospheres alone, at a density equal to that used in the cocultures (total of 300,000 cells/well), also formed neurons at a frequency of $\sim 8 \%$ (Fig. $1 C$ ), this increased neurogenesis can be attributed to an increased cell density. The number of oligodendrocytes formed from the neurosphere-expanded cells were not significantly affected either by a higher cell density or differentiation in the presence of primary cells (Fig. 1C).

\section{Neurosphere cultures show a maintained potential for striatal neuron differentiation}

We next wanted to study the phenotypic identity of the neurons derived from the neurosphere-expanded LGE cells after differentiation in coculture with age-matched primary cells from LGE. Hence, after $7 \mathrm{~d}$ of differentiation (in coculture or on PLL in the absence of primary cells as a control), the cultures were fixed and stained with antibodies against GFP in combination with antibodies detecting ISL1 (striatal projection neuron progenitors/ immature striatal projection neurons), DARPP-32 (striatal projection neurons), or Er81 (olfactory bulb progenitors and neurons).

We have previously reported that neurospheres isolated and expanded from E13.5 LGE followed by differentiation for $7 \mathrm{~d}$ on PLL maintain many of their LGE characteristics, but are more similar to olfactory bulb interneurons because they maintain the expression of Er81 but do not express ISL1 or DARPP-32 (Parmar et al., 2002). These results were confirmed in the present study, in which we found Er81 expression both in neurospheres differentiated in the presence (Fig. $1 D-F$, arrow) or absence of

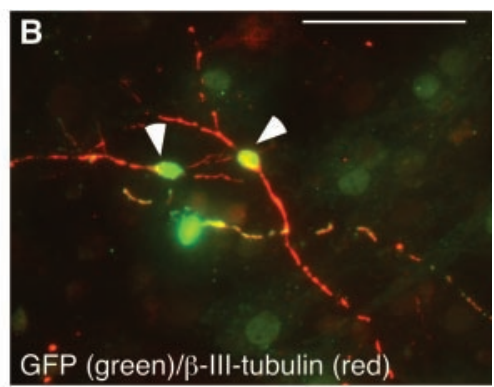

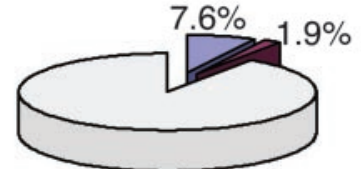

High density neurospheres

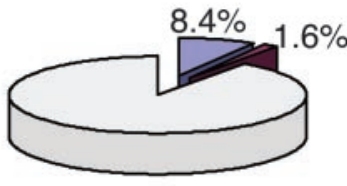

Neurospheres in co-culture neurons aligodendrocytes

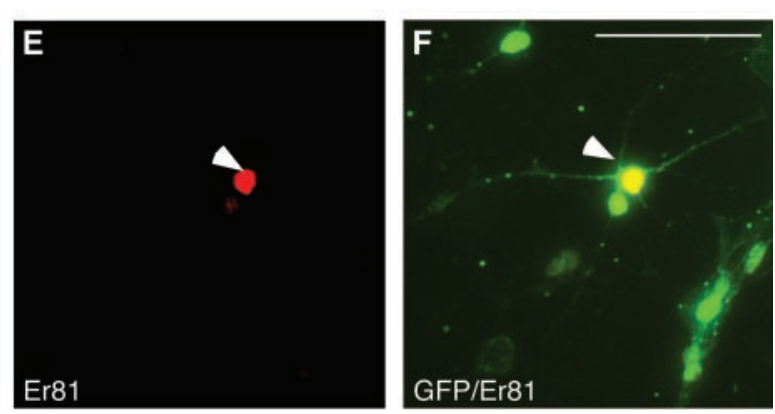

Figure 1. Differentiation of neurosphere-expanded LGE cells in coculture with primary LGE cells does not effect neuron and oligodendrocyte differentiation. $A, B, D-F$, Neurosphere-expanded LGE-derived cells differentiated in coculture with agematched primary LGE cells give rise to $\beta$-III-tubulin + and Er81+ neurons (arrowheads) and cells with glia-like morphologies in coculture with primary LGE cells did not change the percentage of $\beta$-III+ neurons or CNPase + oligodendrocytes generated from the neurospheres. Scale bars, $50 \mu \mathrm{m}$.

primary cells (data not shown). Likewise, no expression of ISL1 could be detected when the neurospheres were differentiated in the absence of primary cells (data not shown). Interestingly, after differentiation in the presence of primary cells, a significant number of the neurosphere-expanded LGE cells (from the transgenic embryos) as detected by GFP immunocytochemistry coexpressed ISL1 (Fig. $2 A-C$ ). Furthermore, a small but significant fraction of the GFP-expressing neurosphere-derived cells with neuronal morphologies, were found to coexpress DARPP-32 (Fig. 2D-F) when differentiated in coculture with primary LGE cells. The ability of neurosphere-expanded LGE cells to differentiate into cells with characteristics of striatal projection neurons was confirmed with retrovirally GFP-labeled neurospheres derived from the LGE of wild-type mice. Again, we detected ISL1-expressing (Fig. 2G-I) and DARPP-32-expressing (Fig. $2 J-L$ ) cells of sphere origin when the neurospheres had been differentiated in coculture with primary LGE cells.

Quantifying the number of neurons with characteristics of olfactory bulb interneurons (as detected by Er81 expression) and the number of neurons with characteristics of striatal projection neurons (as detected by ISL1 expression) before and after coculturing showed that the number of Er81-expressing neurons of sphere origin were not affected by cell density or coculturing: $13 \pm 5 \%$ of the neurons in low-density neurosphere-only cultures, $12 \pm 1 \%$ in high-density neurosphere-alone cultures, and 

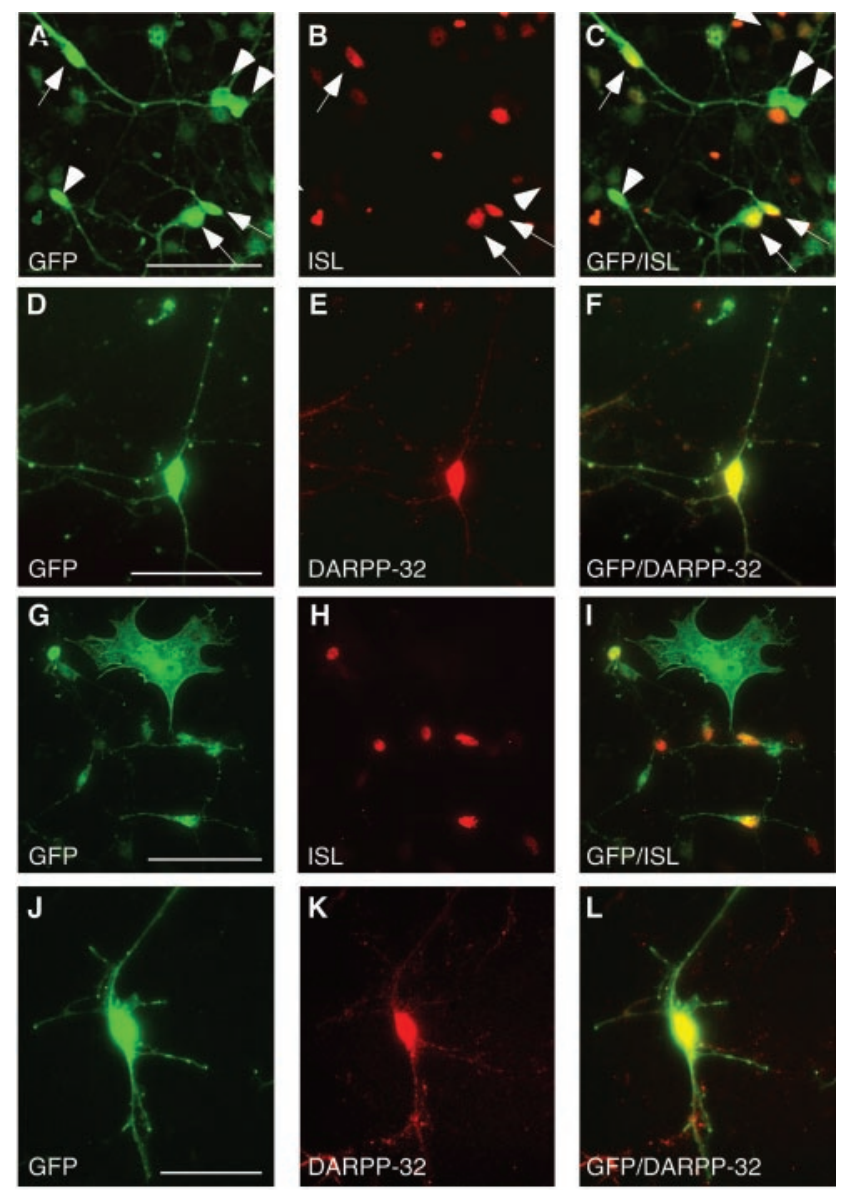

M

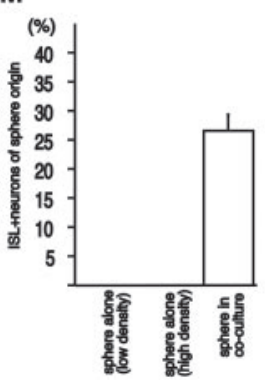

N
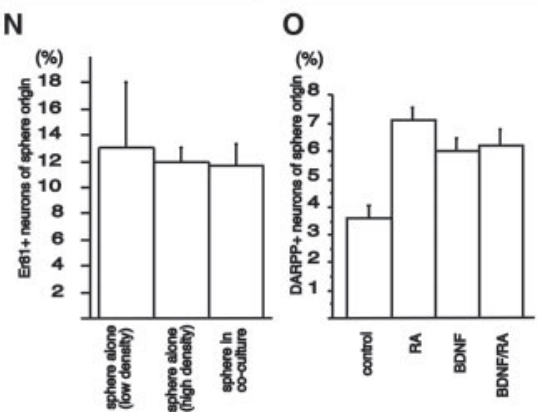

Figure 2. Striatal neuron differentiation of LGE-derived neurospheres differentiated in c0culture with age-matched primary LGE cells. $A-C$, Neurospheres originating from LGE of GFPexpressing transgenic embryos, differentiated into ISL1-expressing cells when differentiated in coculture with primary LGE cells, as detected by coexpression of ISL1 and GFP (arrows). Arrowheads show neurosphere-derived cells not expressing ISL1. D-F, Some neurosphere-derived cells also expressed DARPP-32. G-L, Expression of ISL1 and DARPP-32 in LGE-derived neurospheres was confirmed using neurospheres generated from wild-type mice and labeled with a GFP-expressing retrovirus. $M, N$, Neurosphere-derived ISL1+ cells were not generated at the expense of Er81 + neurons, because differentiation in coculture with primary LGE cells induced the expression of ISL1 but did not change the percentage of neurosphere-derived Er81+ neurons. O, RA and BDNF increase the number of DARPP-32 expressing neurons in the cocultures. Error bars indicate SEM. Scale bars, $50 \mu \mathrm{m}$.

$11.7 \pm 1.7 \%$ in coculture-expressed Er81 (Fig. $2 N$ ). No cells in the neurosphere-alone cultures expressed ISL1, but after differentiation in coculture, $26 \pm 3 \%$ of the neurosphere-derived neurons expressed ISL1 (Fig. $2 \mathrm{M}$ ). Because the proportion of olfactory bulb-like neurons were not affected by the coculture differentiation, the differentiation of cells toward a striatal projection neuron fate does not seem to be at the expense of the Er81-expressing population of neurons.
BDNF, RA, SHH, and NT-3 are not sufficient to induce striatal neurons from neurosphere-expanded LGE cells, but enhance the number of DARPP-32-expressing neurons generated in coculture with primary cells

Factors such as RA, BDNF, SHH, and NT-3 have been shown to increase the number of ISL1 and/or DARPP-32-expressing cells in telencephalic and spinal cord organotypic slice and primary cultures (Roelink et al., 1995; Ivkovic et al., 1997; Kohtz et al., 1998, 2001; Dutton et al., 1999a,b; Ivkovic and Ehrlich, 1999; Toresson et al., 1999). To determine whether any of these proteins were the factor or factors produced in the primary cultures responsible for the induction of ISL1 and DARPP-32 in the expanded cell, we differentiated the LGE-derived neurospheres in the presence of BDNF, RA, SHH, NT-3, or in a combination of either BDNF and RA or SHH and NT-3, at concentrations previously reported to have optimal effects in vitro (see Materials and Methods).

After differentiation of LGE neurospheres in the presence of BDNF and/or RA, the proportion of neurons generated was not significantly different, and in all differentiation conditions, the majority of neurons were GABAergic (supplemental Fig. 1, available at www.jneurosci.org/cgi/content/full/24/31/6958/DC1). The proportion of GABAergic cells increased significantly $(p<$ 0.05 ), from $\sim 65 \%$ of the neurons under standard conditions, to $\sim 100 \%$ of the neurons when the neurospheres had been differentiated in BDNF and RA combined (supplemental Fig. 1, available at www.jneurosci.org/cgi/content/full/24/31/6958/DC1). However, in contrast to the reported effect on DARPP-32 expression in primary cultures (Ivkovic et al., 1997; Ivkovic and Ehrlich, 1999; Toresson et al., 1999), under none of these differentiating conditions did we detect any ISL1- or DARPP-32-expressing cells in the differentiated neurosphere cultures (data not shown). Likewise, $\mathrm{SHH}$ alone, or in combination with NT-3, has been shown to increase the number of ISL1-expressing cells in telencephalic and spinal cord organotypic slice cultures (Roelink et al., 1995; Kohtz et al., 1998, 2001; Dutton et al., 1999a,b). However, we never detected any ISL-expressing cells when adding various concentrations of $\mathrm{SHH}$ alone, or in combination with NT-3 (see Materials and Methods for details), during differentiation of LGE-derived neurospheres (data not shown).

Next, we wanted to see whether RA and/or BDNF had an effect on the number of DARPP-32-expressing neurons generated from neurosphere-expanded LGE cells that were differentiated in coculture with age-matched primary cells (a condition that is sufficient to promote ISL1 and DARPP-32 expression as shown above). Here, we could see a clear effect of RA and BDNF. Addition of either factor to the medium resulted in an approximately twofold increase of neurosphere-derived DARPP-32expressing neurons (Fig. 2O).

\section{Region-specific effects on striatal neuron differentiation}

We next wanted to determine whether the observed effects on striatal neuron differentiation of expanded LGE cells were region specific, such that the primary LGE cells, but not primary cells from other regions of the developing brain, could support differentiation of neurons with a striatal projection neuron-like phenotype. Thus, LGE-derived neurospheres were differentiated in coculture with primary cells taken either from the E13.5 LGE or from two other forebrain regions: the MGE and developing CTX or from the E12.5 VM. Approximately the same percentage of the GFP-positive cells with neuronal morphologies were detected when the neurosphere-expanded LGE cells were differentiated in 
coculture with cells from the MGE (18 $\pm 4.5 \%)$ and VM (20 \pm $1.9 \%)$, as from the LGE $(13 \pm 2.1 \%)$. However, when differentiated in the presence of primary cortical cells, significantly more GFP-positive cells with neuronal morphologies $(42 \pm 5.8 \%)$ were detected (Fig. $3 A$ ). We found that primary LGE cells were the most efficient in promoting ISL1 expression (Fig. 3B): $28 \pm$ $5 \%$ of the GFP-expressing expanded cells with neuronal morphologies expressed ISL1 when cocultured with primary LGE cells (Fig. $2 A-C, G, H$ ), compared with $6 \pm 2 \%$ when differentiated in coculture with primary MGE cells (Fig. $3 B$ ). Although an equal number of GFP-positive cells with neuronal morphologies could be detected when LGE cells were differentiated on top of primary cells from the VM (Fig. $3 A$ ) and more neurons could be detected when differentiated on top of primary cells from the cortex (Fig. 3A), ISL1 expression was never detected when the neurosphere-expanded LGE cells were differentiated in coculture with primary cortical or VM cells (Fig. 3B).

When analyzing the cultures further, we noticed that the primary cortical cells not only promoted neuronal differentiation, but also affected the morphology of the neurosphere-expanded LGE cells. After $3 \mathrm{~d}$ of differentiated in coculture with primary LGE or cortical cells, most of the neurosphere-derived cells (detected by retroviral expression of GFP) displayed similar immature neuronal morphologies (Fig. 3C,E). However, after $7 \mathrm{~d}$ in coculture with primary LGE cells, the majority of the neurosphere-derived cells that expressed GFP had the morphology of astrocytes (Fig. 3D). When the same LGE-derived neurospheres were differentiated in coculture with primary cells from E13.5 CTX for $7 \mathrm{~d}$, many of the GFP-expressing cells displayed mature neuronal morphologies with elaborate processes (Fig. $3 F$ ).

Such effects on the morphology of the LGE-derived cells was not evident when neurosphere-expanded LGE-derived cells were differentiated for $7 \mathrm{~d}$ in coculture with primary cells from E12.5 VM (Fig. 3G), and although many of the primary VM cells expressed tyrosine hydroxylase $(\mathrm{TH})$, none of the expanded cells were found to express TH (Fig. 3G). From the present study it is difficult to determine whether the failure to generate $\mathrm{TH}$ expressing neurons from LGE neurospheres is attributable to irreversible forebrain specification of the expanded cells or lack of guiding cues in the VM primary culture.

Taken together, these data show that the neurosphereexpanded LGE cells maintain the potential to differentiate into neurons with characteristics of striatal projection neurons when provided with appropriate cues. The factor or factors provided by the primary cells seem to be region specific, because only primary LGE and to some extent primary MGE, but not primary cortical and VM cells were found to provide the factors necessary to guide striatal projection neuron differentiation.

Striatal neuron differentiation of neurosphere-expanded cells require direct cell-to-cell contact with primary cells

To investigate the nature of the factor or factors in the primary LGE cultures responsible for promoting a striatal phenotype of the neurosphere-expanded cells, we used two different assays in which the neurosphere-expanded cells were differentiated in medium taken from cultures of primary cells, but in which direct cell-to-cell contact was prevented.

In the first assay, expanded cells were differentiated in CM obtained from primary LGE cells grown in parallel. Fifty percent of the medium was removed from the primary cultures (referred to as $\mathrm{CM}$ ) and added to the differentiating neurospheres every 48 $\mathrm{hr}$ for $7 \mathrm{~d}$. To ensure that the concentration of the striatalpromoting factor or factors in this $\mathrm{CM}$ was sufficient to promote
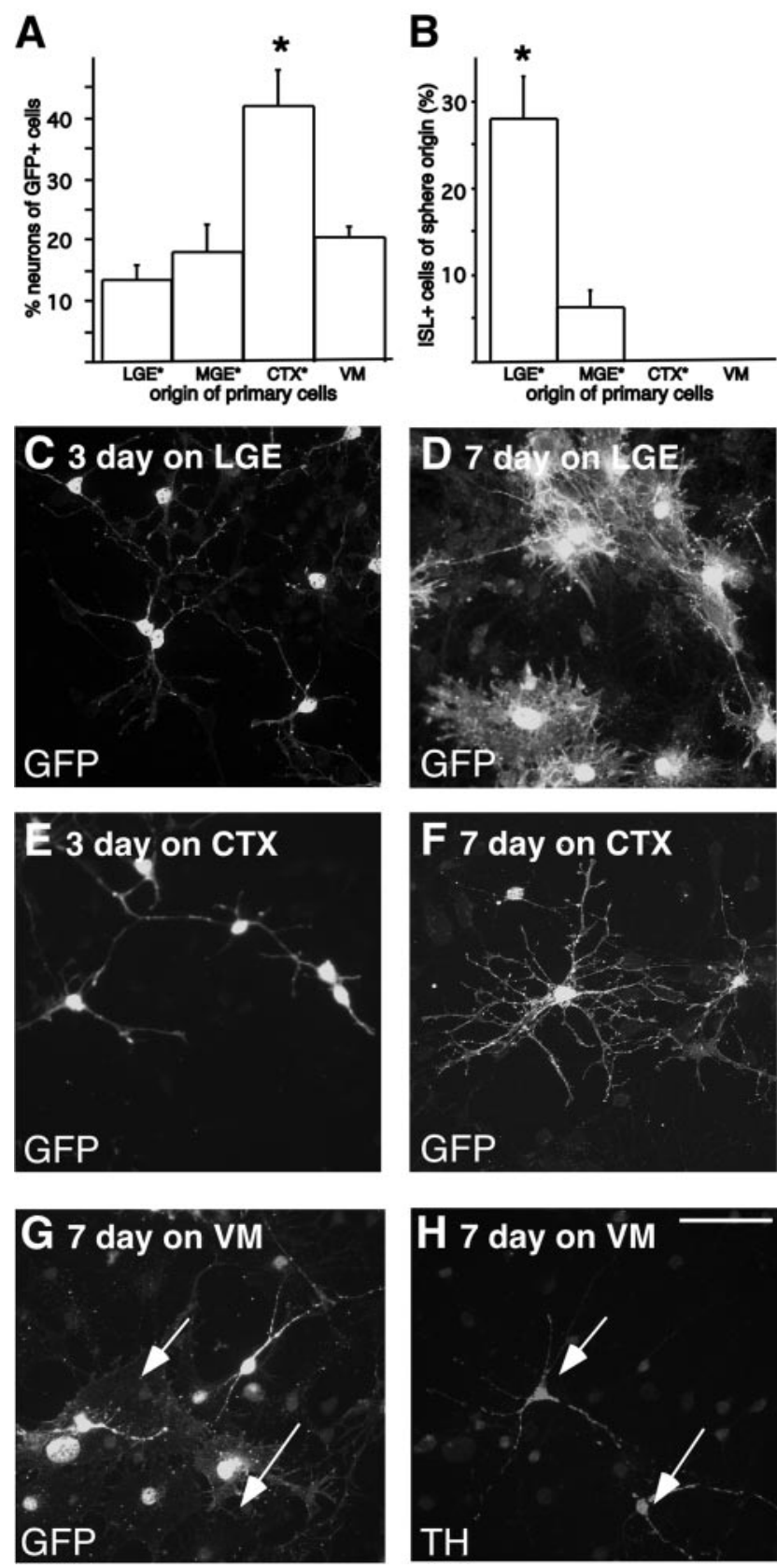

Figure 3. Region-specific effects on striatal neuron differentiation. $A, B$, The extent of striatal neuron differentiation of expanded LGE cells was estimated by quantifying the percentage of neurosphere-derived GFP-expressing neurons that also expressed ISL1. Primary LGE cells were found to be the most efficient in inducing the expression of ISL1 in the neurosphere-expanded cells. C, E, When analyzing the morphology of the neurosphere-derived cells, the majority of the GFP-expressing cells had immature neuronal morphologies after $3 \mathrm{~d}$ of coculturing on primary LGE and primary cortical cells. D, F, After $7 \mathrm{~d}$ in coculture, the majority of the GFP-expressing cells differentiated with primary LGE cells had the morphology of astrocytes, in contrast to when they were differentiated on cortical cells, where the majority of GFP + cells displayed mature neuronal morphologies. G, Primary cells from the VM did not affect the morphology of the expanded cells, and although many of the primary cells expressed TH (arrows), TH expression was never detected in the expanded cells. Error bars indicate SEM. Scale bar, $50 \mu \mathrm{m}$. All neurospherederived cells were identified using retroviral GFP labeling, except cultures marked with an asterisk, which were derived from transgenic GFP-expressing embryos.

striatal neuron differentiation from the expanded cells, a positive control was always processed in parallel. In these control cultures, $50 \%$ of the medium in a standard coculture assay (primary cells and expanded cells in the same well) was renewed every $48 \mathrm{hr}$, 

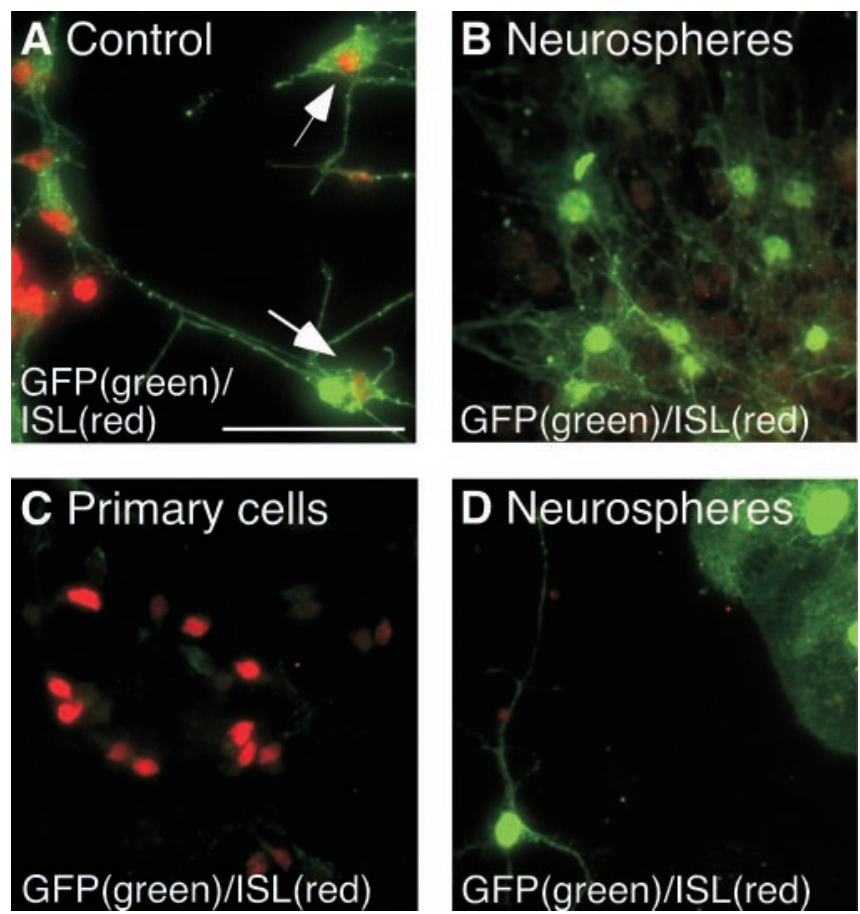

Figure 4. Soluble factors from primary cultures are insufficient in promoting ISL1. $A, B$, Condition-medium (CM) assay: neurosphere-expanded LGE cells express ISL1 when in contact with primary LGE cells during differentiation (control), but when differentiated in CM obtained from the same primary cells, no expression of ISL 1 could be detected in the expanded cells. C, D, Shared medium assay: when primary LGE cells and neurosphere-expanded LGE cells were differentiated in separate wells but with shared medium, ISL could be detected in the primary cells, but not in the expanded cells. Scale bar, $50 \mu \mathrm{m}$.

thus mimicking the conditions in the condition medium assay. In these control cultures, we detected ISL1-expressing cells of sphere origin (Fig. 4A), indicating that the factor was present in sufficient quantity. In the neurosphere cultures differentiated in the same concentration of CM, however, we never detected any ISL1expressing cells (Fig. $4 B$ ).

We also performed an assay in which the two types of cells are cultured separately, but in the same medium, in a two-chamber system. In this assay, the primary and expanded cells are initially plated in wells separated by a low wall. When the cells have attached, the level of the medium is raised, so that the medium is shared but cell mixing is prevented. When cultured in this manner, many of the primary cells express ISL1 (Fig. 4C), but no ISL1 expression in the neurosphere-expanded cells could be detected (Fig. 4D). This lack of striatal neuron differentiation is not caused by insufficient concentration of striatal promoting factors in the shared medium, because ISL1 expression was detected in a control culture, in which expanded cells were differentiated in the same well as the primary LGE cells sharing medium with an empty well (data not shown).

Both of these results indicate that the factor or factors necessary to guide striatal neuron differentiation from the neurosphereexpanded cells are not soluble i.e., freely diffusible in the medium, and direct contact between the primary and expanded cells is therefore likely to be required.

\section{Monolayer-expanded cells do not differentiate into striatal neurons}

We next asked whether the signals present in the primary LGE cultures could guide LGE cells expanded as attached monolayer culture to differentiate into neurons expressing markers characteristic of striatal projection neurons. We performed the same coculture differentiation paradigm on E13.5 LGE cells isolated from TgR ROSA26 (ROSA 26) mice (Friedrich and Soriano, 1991) and expanded as adherent cultures in 10\% serum and EGF. Cells from the ROSA26 mouse ubiquitously express LacZ and have previously been used in CNS grafting experiments (Olsson et al., 1997). The transgene is known to be maintained in DARPP32-expressing cells after transplantation (K. Campbell, personal communication). The expanded cells (passage 5) were plated under the same coculture differentiation conditions as the neurospheres (1\% serum, growth factor free), on top of primary LGE cells and were allowed to differentiate for 7 or $10 \mathrm{~d}$. After fixation, the expanded cells were detected using an antibody directed against $\beta$-galactosidase ( $\beta$-gal). $\beta$-gal was predominantly located in the cytoplasm, and cells with both glia-like and neuron-like morphologies could be detected. Many of the cells with neuronlike morphologies were found to coexpress $\beta$-III-tubulin (supplemental Fig. 2, available at www.jneurosci.org/cgi/content/full/ 24/31/6958/DC1). However, despite a significant number of primary cells expressing ISL1, we never detected any of the $\beta$-galexpressing expanded cells that coexpressed ISL1 (supplemental Fig. 2, available at www.jneurosci.org/cgi/content/full/24/31/ 6958/DC1). Even after $10 \mathrm{~d}$ of differentiation in coculture with primary LGE cells, none of the monolayer-expanded were found to coexpress ISL1 or DARPP-32 (data not shown).

The observed striatal neuron differentiation in neurosphereexpanded LGE cells depends on the expression of Gsh2

One of the major differences between LGE cells expanded as monolayer or neurosphere cultures is that the Gsh2-expressing progenitors are maintained only in the neurosphere cultures (Parmar et al., 2002; Skogh et al., 2003). To examine whether the potential for striatal neuron differentiation in neurosphereexpanded cells depends on the presence of these Gsh2-expressing progenitors, we made use of genetically modified mice lacking Gsh2 (Szucsik et al., 1997). Neurospheres were generated from the LGE of Gsh2 heterozygous ( $+/-)$ and homozygous ( $-/-$ ) littermates at E12 (before Gsh1 shifts up and compensates for the lack of Gsh2). The Gsh2-/- neurospheres showed the same growth profile as the Gsh $2+/-$ neurospheres, with a constant growth from passage 2 and onward. However, the Gsh2-deficient neurospheres showed a somewhat lower rate of expansion than the Gsh $2+/-$ cells (Fig. 5A). The Gsh $2+/-$ and Gsh $2-/-$ cells generated the same number of secondary neurospheres at low ( 5 cells/ $\mu \mathrm{l}$ ) densities, whereas at higher densities the number of neurospheres was slightly lower in the Gsh2-/- cultures compared with the Gsh2+/- cultures (Fig. $5 B$ ). At both high and low densities, the Gsh2-I- neurospheres were significantly smaller ( $p<$ 0.05) than the Gsh2+/- neurospheres (Fig. 5C). Because the individual cells within each sphere are similar in size between the Gsh2-1- and Gsh2+/- cultures, we believe that this reflects the proliferation defect previously described for the Gsh2deficient mice (Toresson et al., 2000; Toresson and Campbell, 2001). We also noticed a small (but not significant, $p>0.05$ ) decrease in the percentage of neurons generated after differentiation of the neurospheres lacking Gsh2, compared with Gsh2+/- control cultures (Fig. 5D). The neurons that formed from the Gsh2-negative neurospheres appeared to have a similar morphology to those generated from wild-type neurospheres and matured into a GABAergic phenotype. However, the proportion of GABAergic neurons were slightly lower in the Gsh2-/cultures than in the control cultures: $\sim 40 \%$ of the neurons in 


\section{A. Growth curve}

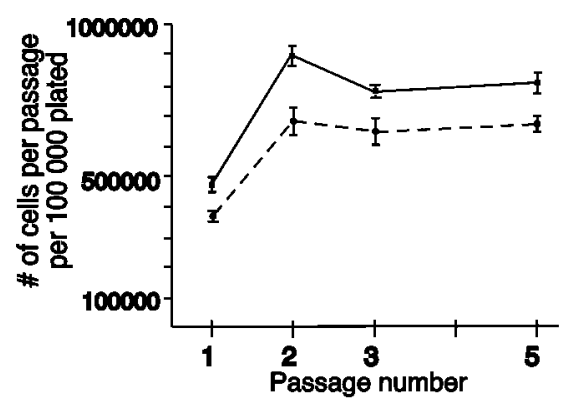

B. Sphere-forming assay

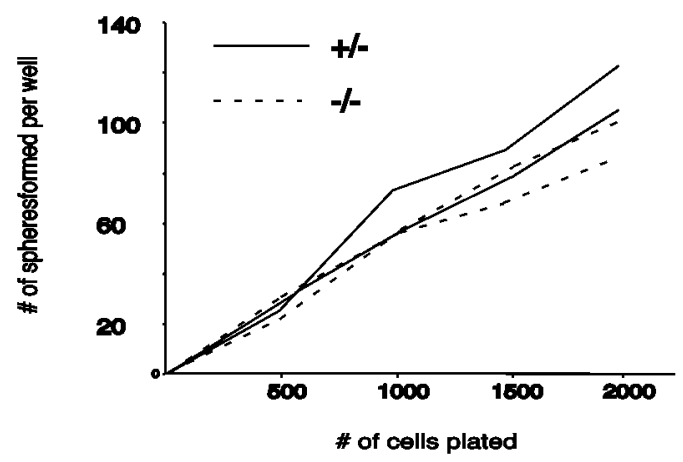

C. Sphere size

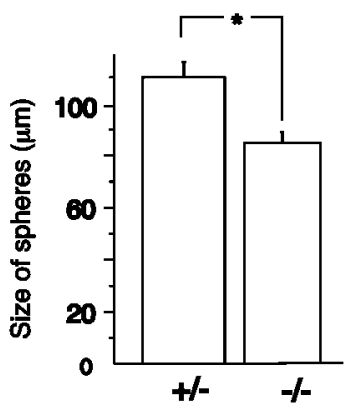

D. Neuronal differentiation

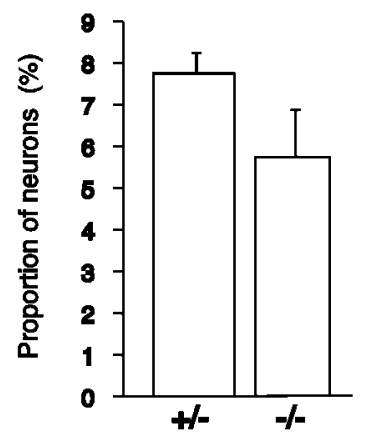

Figure 5. Proliferation and sphere-forming abilities of Gsh2-/- cells. $A$, Neurospheres generated from the LGE of E12 Gsh2 + I - and - / - littermates showed the same growth profile, but the $-1-$ cultures had a somewhat lower rate of expansion compared with $+1-$ cultures. $B$, The ability to form secondary spheres were only slightly compromised in the $-1-$ cultures, and only at high densities. $C$, However, the $-I-$ neurospheres were significantly $(p<0.05)$ smaller in size than the $+/-$ neurospheres. $D$, The percentage of neurons generated after differentiation was not significantly different in the $-/$ - cultures compared with the $+/-$ cultures. Error bars indicate SEM. ${ }^{*} p<0.05$.

the Gsh2-I- cultures were GABAergic, compared with $\sim 65 \%$ of the neurons in wild-type control cultures. The olfactory bulb progenitors and neurons (as detected by Er81 expression) were not affected in cultures established from Gsh2-/- mice. Er81 expression was maintained in differentiated cultures lacking Gsh2, at the same frequency $(\sim 12 \%)$ as in wild-type cultures.

To evaluate the striatal neuron differentiation potential of neurosphere-expanded LGE cells lacking Gsh2, the expanded cells were marked by GFP using the same retrovirus and infection protocol as described for the wild-type E13.5 LGE. Both E13.5 wild-type and E12 heterozygous cultures were processed in parallel as positive controls. The retroviral infection resulted in ap- proximately the same number of GFP-expressing cells in all cultures, and in all cases both GFP-expressing neurons and glia were observed. We found that a portion of the cells originating from Gsh2+/- neurospheres started to express ISL1 when these cells were differentiated in coculture with primary LGE cells (Fig. 6A$C$ ), just like the E13.5 wild-type LGE cells (as seen in Fig. 2G-I). When quantifying the number of GFP-expressing cells with neuronal morphology expressing ISL1 in the heterozygous and wildtype control cultures, we found no difference in the proportion of ISL1-expressing cells of sphere origin between the two types of cultures ( $26 \pm 3$ and $32 \pm 7 \%$, respectively) (Fig. $6 H$ ). In contrast, although comparable (slightly lower but not significantly different) numbers of the expanded Gsh2-/- cells differentiated into cells with neuronal morphologies (Fig. 6G), we never detected any GFP-expressing cells that also expressed ISL1 when coculturing the neurospheres lacking Gsh2 with primary LGE cells for $7 \mathrm{~d}$ (Fig. 6D-F,H).

In summary, LGE cells derived from Gsh2-/- mice can be expanded as neurospheres with only slight changes in proliferation and neurosphere-forming abilities, whereas their potential to differentiate into neurons with a striatal phenotype is compromised.

\section{Discussion}

We report here a culture system, in which expanded neural stem and progenitor cells are differentiated in coculture with primary cells. We show that the primary cells provide contact-mediated region-specific developmental cues to the expanded cells and that these cues are sufficient to guide the expanded LGE cells toward a striatal neuron fate. We also show that the ability of the expanded LGE cells to respond to the cues presented by the primary LGE cells depends on the maintenance of Gsh2 expression in the expanded cells. Based on our results, we suggest that the Gsh2expressing cells represent the principal striatal projection neuron progenitors in expanded LGE cultures and that maintenance of this population is crucial for maintaining a capacity for striatal neuron differentiation after expansion.

\section{Maintained potential for striatal neuron differentiation in neurosphere-expanded LGE cells}

In this study, we used growth factor-expanded neural stem and progenitor cells from the LGE, a structure that during embryonic development, generates both the striatal projection neurons and the interneurons of the olfactory bulb (Deacon et al., 1994; Olsson et al., 1998; Wichterle et al., 1999). We have previously reported that although many LGE characteristics are maintained in neurosphere cultures in vitro, the ISL1-expressing striatal progenitor population is not present in these cultures (Parmar et al., 2002). This suggests that the expanded neurosphere cultures lack specified progenitors for striatal projection neurons. Accordingly, when the expanded cells are differentiated in vitro, no DARPP-32-expressing neurons are generated. However, the expression of Gsh2, a homeodomain transcription factor involved in the specification of both striatal projection neurons and olfactory bulb interneurons in vivo (Corbin et al., 2000; Toresson et al., 2000; Toresson and Campbell, 2001; Yun et al., 2001), is maintained and translated into protein in LGE-derived neurospheres (Parmar et al., 2002), suggesting that the cells have a latent potential for striatal neuron differentiation. In this study, we have shown that LGE cells expanded as neurospheres and differentiated in coculture with primary LGE cells start to express ISL1, and subsequently mature into DARPP-32-expressing neurons. A similar differentiation potential of neurosphereexpanded LGE cells has also been reported in transplantation 

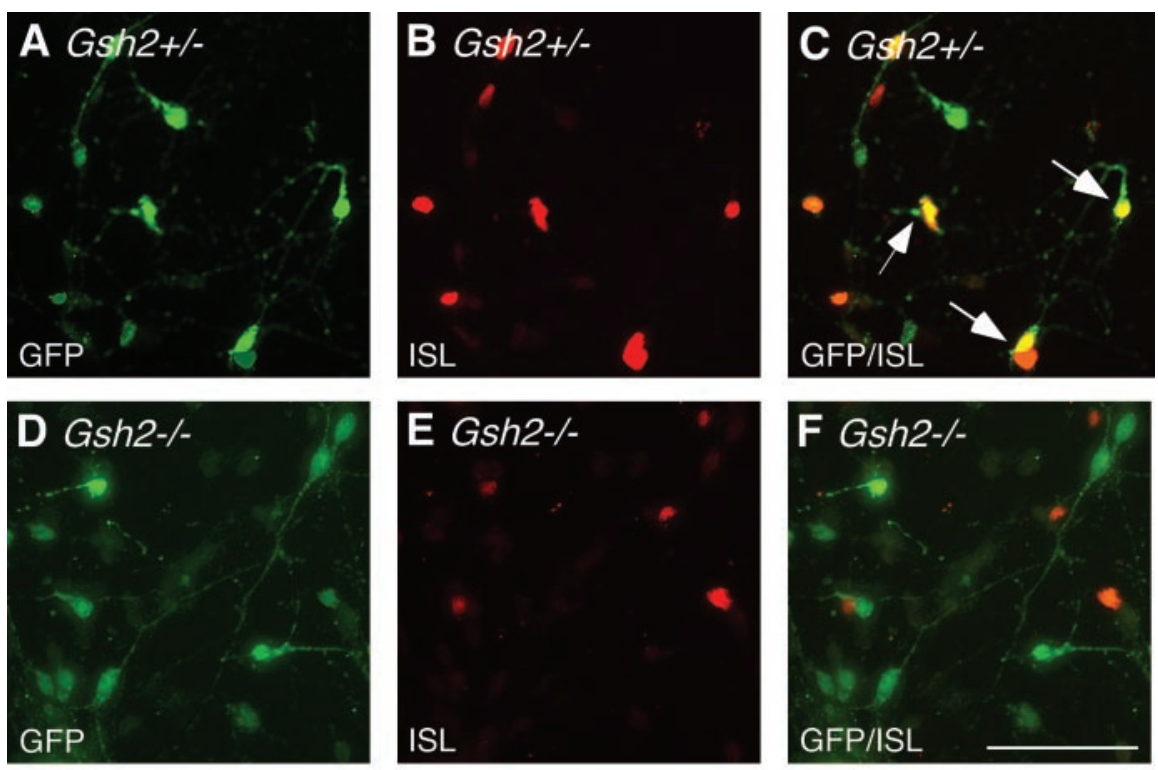

G

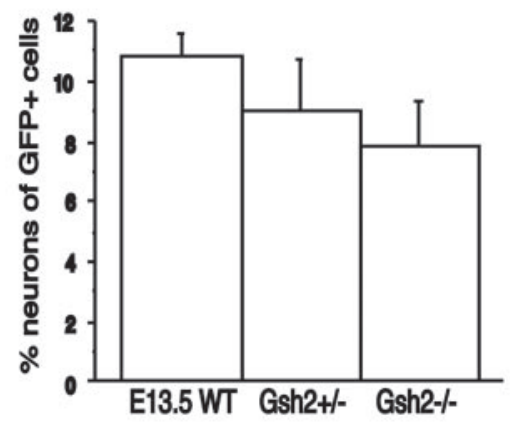

H $(\%)$

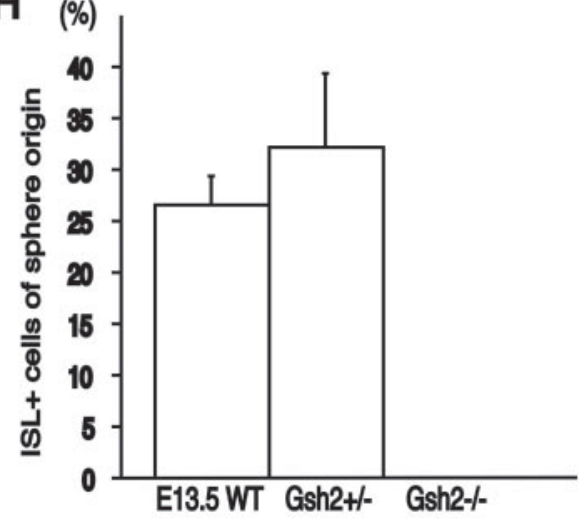

Figure 6. Striatal neuron differentiation depends on Gsh2 expression. $A-C, H$, Cells in Gsh2+/--derived neurospheres express ISL1, at a level indistinguishable from wild-type neurospheres, when differentiated in coculture with primary LGE cells. $D-F, H$, However, the ability to express ISL1 when differentiated in coculture with primary cells was lost in the Gsh2-/--derived neurospheres. G, The numbers of GFP + cells with neuronal morphologies were comparable in wild-type, Gsh2+/-, and Gsh2-/- cultures. Error bars indicate SEM. Scale bar, $50 \mu \mathrm{m}$.

studies, in which a few cells derived from LGE-forebrain neurospheres were shown to express DARPP-32 after transplantation to the striatum of neonatal or adult rats (Fricker et al., 1999; Eriksson et al., 2003). Taken together, these results show that striatal progenitors are expandable in neurosphere cultures, but need to be presented with specific developmental cues to differentiate into neurons with characteristics of striatal projection neurons.

\section{Nature of the striatal promoting factor or factors}

It has been shown that LGE-derived signals may be necessary for striatal neuron differentiation to occur in vitro (this study) and after transplantation (Magrassi et al., 1998). Our analysis also showed that the factor or factors provided by the primary cells are mediated, at least in part, by direct cell-to-cell contact. Furthermore, the striatal-promoting effect seems to be region specific: differentiation in coculture with cells from all areas allows for neuronal differentiation, yet primary LGE cells, and to a lesser extent primary MGE cells, but not primary cells from other areas of the developing brain, could promote the differentiation of cells with characteristics of developing and mature striatal projection neurons.

The Gsh2-expressing cell is the principal striatal progenitor present in LGE neurosphere cultures

In contrast to primary LGE cells in which $\mathrm{RA}, \mathrm{BDNF}$, and SHH can promote the differentiation of striatal projection neurons in vitro (Ivkovic et al., 1997; Kohtz et al., 1998; Toresson et al., 1999), treatment of neurosphere-expanded LGE cells with these factors during differentiation did not induce any striatal projection neuron differentiation. We suggest that this is caused by differences in maturation status of the striatal progenitors present in the two types of cultures. In contrast to primary cell cultures from the embryonic LGE, which contains not only the immature GSH2-expressing cell population, but also an intermediate, more specified progenitor population, equivalent to the ISL1 expressing SVZ population, the cultures of neurosphere-expanded LGE cells only contains the earlier GSH2-expressing population. It seems likely, therefore, that the factors examined promotes the formation of DARPP-32-expressing striatal neurons by acting on the intermediate progenitors present in the primary cultures but lacking in the neurosphere cultures. Thus, RA and BDNF increase the number of striatal neurons generated from neurosphereexpanded cells only when these are differentiated in coculture with primary cells, a condition in which we have shown that the intermediate progenitor population is present.

We therefore conclude that as yet unidentified additional factors (present in the cocultures) are likely to be required to guide striatal neuron differentiation from the immature GSH2-expressing cells, which are likely to constitute the principal striatal neuron progenitor population present in the long-term expanded LGE neurosphere cultures.

\section{Gsh2 expression is required for differentiation of striatal neuron from expanded LGE cells}

Genetic studies show that Gsh2 is important for the generation of both striatal projection neurons and olfactory bulb interneurons, because both of these populations and their progenitors are compromised in mice lacking Gsh2, and in double mutants lacking both functional Gsh1 and Gsh2 (Toresson et al., 2000; Toresson and Campbell, 2001; Yun et al., 2001; Stenman et al., 2003). In the adult brain, Gsh2 expression is maintained in the SVZ (Parmar et al., 2003b), where the olfactory bulb neurons are generated postnatally (Alvarez-Buylla and Garcia-Verdugo, 2002). Recent studies show that cells in the SVZ also, under experimental conditions, can generate striatal projection neurons (Arvidsson et al., 2002; Parent et al., 2002; Chmielnicki et al., 2004). Thus, Gsh2expressing cells may be involved in the generation of both striatal and olfactory neurons also in the adult brain. 
In previous in vitro studies, we have shown that Er81, a marker for olfactory bulb progenitors and neurons (Stenman et al., 2003) is maintained in neurosphere cultures (Parmar et al., 2002), and we now show, that given the right developmental cues, striatal neuron differentiation also occur. Thus, the in vitro expanded Gsh2-expressing cells are likely to have maintained the same bipotent differentiation potential as their in vivo counterparts. To analyze the requirement for Gsh2 expression more directly in expanded cells, we performed two separate lines of experiments.

First, we wanted to test whether cues from the developing LGE also could direct striatal neuron differentiation from monolayerexpanded cells. In these monolayer cultures, both the ISL1 and GSH2-expressing progenitor populations are lost during expansion (Skogh et al., 2003), suggesting that the monolayer cultures represent a more restricted progenitor population with limited striatal neuron differentiation potential. In support of this, when human monolayer-expanded LGE cells are transplanted to the neonatal rat brain, they behave like endogenous olfactory bulb progenitors, and they do not differentiate site-specifically into DARPP-32-expressing neurons when placed in the striatal parenchyma (Parmar et al., 2003a). Because striatal neurogenesis is virtually complete by birth (Bayer and Altman, 1995), it is possible that the neonatal striatum lacks some cues that are only present during development in the LGE, and that these cues are needed to direct the monolayer-expanded cells toward a striatal projection neuron fate. However, when we differentiated the monolayer-expanded cells in coculture with primary LGE cells they did not start to express ISL1 or differentiate into DARPP32-expressing neurons. We propose that the inability of the LGEderived monolayer cultures to differentiate into DARPP-32expressing neurons, even in the presence of the primary LGE cells, is attributable to the fact that the Gsh2-expressing progenitors are not expanded in this culture paradigm.

In the second experiment, we cocultured neurospheres originating from the LGE of Gsh2-deficient mice. In wild-type LGE neurospheres, the Gsh2-expressing progenitor population is sustained, and we have shown that they are not restricted to olfactory bulb-like neuronal differentiation, but can be guided toward a striatal projection neuron-like fate via ISL1-expressing progenitors when exposed to the appropriate cues. However, neurospheres generated from mice lacking Gsh2 gene expression did not differentiate into striatal neurons when cocultured with primary cells. These results support our hypothesis that the presence of Gsh2-positive cells is crucial for striatal differentiation of expanded cells.

Thus, we advocate that some of the same transcription factors important for striatal neuron differentiation in vivo, like Gsh2 and Isl1 also is critical for striatal neuron differentiation from expanded cells in vitro.

In conclusion, the coculture differentiation assay presented here provides a useful system for studying fate specification and differentiation potential of expanded neural stem and progenitor cells. In the past, studies addressing similar questions for primary cells have successfully been addressed in transplantation studies (Gaiano and Fishell, 1998; Campbell and Olsson, 2000). Grafting experiments with expanded cells has been less useful for studies of fate specification, because the extent of neuronal differentiation from immature progenitors in the host brain environment is limited (Winkler et al., 1998; Eriksson et al., 2003). This limitation is not seen in the in vitro coculture system. This assay, therefore, provides an attractive alternative model for studying properties of fate specification and differentiation potential of growth factor expanded neural progenitor cells under conditions that can be controlled and manipulated.

\section{References}

Alvarez-Buylla A, Garcia-Verdugo JM (2002) Neurogenesis in adult subventricular zone. J Neurosci 22:629-634.

Arvidsson A, Collin T, Kirik D, Kokaia Z, Lindvall O (2002) Neuronal replacement from endogenous precursors in the adult brain after stroke. Nat Med 8:963-970.

Bayer SA, Altman J (1995) Neurogenesis and neuronal migration. In: The rat nervous system (Paxinos G, ed), pp 1041-1078. San Diego: Academic.

Campbell K, Olsson M (2000) Novel mechanisms in mammalian telencephalic development as revealed by neural transplantation. Prog Brain Res 127:99-113.

Chmielnicki E, Benraiss A, Economides AN, Goldman SA (2004) Adenovirally expressed noggin and brain-derived neurotrophic factor cooperate to induce new medium spiny neurons from resident progenitor cells in the adult striatal ventricular zone. J Neurosci 24:2133-2142.

Corbin JG, Gaiano N, Machold RP, Langston A, Fishell G (2000) The Gsh2 homeodomain gene controls multiple aspects of telencephalic development. Development 127:5007-5020.

Deacon TW, Pakzaban P, Isacson O (1994) The lateral ganglionic eminence is the origin of cells committed to striatal phenotypes: neural transplantation and developmental evidence. Brain Res 668:211-219.

Dutton R, Yamada T, Turnley A, Bartlett PF, Murphy M (1999a) Regulation of spinal motoneuron differentiation by the combined action of Sonic hedgehog and neurotrophin 3. Clin Exp Pharmacol Physiol 26:746-748.

Dutton R, Yamada T, Turnley A, Bartlett PF, Murphy M (1999b) Sonic hedgehog promotes neuronal differentiation of murine spinal cord precursors and collaborates with neurotrophin 3 to induce Islet-1. J Neurosci 19:2601-2608.

Eriksson C, Bjorklund A, Wictorin K (2003) Neuronal differentiation following transplantation of expanded mouse neurosphere cultures derived from different embryonic forebrain regions. Exp Neurol 184:615-635.

Fricker RA, Carpenter MK, Winkler C, Greco C, Gates MA, Bjorklund A (1999) Site-specific migration and neuronal differentiation of human neural progenitor cells after transplantation in the adult rat brain. J Neurosci 19:5990-6005.

Friedrich G, Soriano P (1991) Promoter traps in embryonic stem cells: a genetic screen to identify and mutate developmental genes in mice. Genes Dev 5:1513-1523.

Gaiano N, Fishell G (1998) Transplantation as a tool to study progenitors within the vertebrate nervous system. J Neurobiol 36:152-161.

Hadjantonakis AK, Gertsenstein M, Ikawa M, Okabe M, Nagy A (1998) Generating green fluorescent mice by germline transmission of green fluorescent ES cells. Mech Dev 76:79-90.

Hitoshi S, Tropepe V, Ekker M, van der Kooy D (2002) Neural stem cell lineages are regionally specified, but not committed, within distinct compartments of the developing brain. Development 129:233-244.

Ivkovic S, Ehrlich ME (1999) Expression of the striatal DARPP-32/ ARPP-21 phenotype in GABAergic neurons requires neurotrophins in vivo and in vitro. J Neurosci 19:5409-5419.

Ivkovic S, Polonskaia O, Farinas I, Ehrlich ME (1997) Brain-derived neurotrophic factor regulates maturation of the DARPP-32 phenotype in striatal medium spiny neurons: studies in vivo and in vitro. Neuroscience 79:509-516.

Kohtz JD, Baker DP, Corte G, Fishell G (1998) Regionalization within the mammalian telencephalon is mediated by changes in responsiveness to sonic hedgehog. Development 125:5079-5089.

Kohtz JD, Lee HY, Gaiano N, Segal J, Ng E, Larson T, Baker DP, Garber EA, Williams KP, Fishell G (2001) N-terminal fatty-acylation of sonic hedgehog enhances the induction of rodent ventral forebrain neurons. Development 128:2351-2363.

Magrassi L, Ehrlich ME, Butti G, Pezzotta S, Govoni S, Cattaneo E (1998) Basal ganglia precursors found in aggregates following embryonic transplantation adopt a striatal phenotype in heterotopic locations. Development 125:2847-2855

Okabe M, Ikawa M, Kominami K, Nakanishi T, Nishimune Y (1997) "Green mice" as a source of ubiquitous green cells. FEBS Lett 407:313-319.

Olsson M, Campbell K, Wictorin K, Bjorklund A (1995) Projection neurons 
in fetal striatal transplants are predominantly derived from the lateral ganglionic eminence. Neuroscience 69:1169-1182.

Olsson M, Campbell K, Turnbull DH (1997) Specification of mouse telencephalic and mid-hindbrain progenitors following heterotopic ultrasound-guided embryonic transplantation. Neuron 19:761-772.

Olsson M, Bjorklund A, Campbell K (1998) Early specification of striatal projection neurons and interneuronal subtypes in the lateral and medial ganglionic eminence. Neuroscience 84:867-876.

Ory DS, Neugeboren BA, Mulligan RC (1996) A stable human-derived packaging cell line for production of high titer retrovirus/vesicular stomatitis virus G pseudotypes. Proc Natl Acad Sci USA 93:11400-11406.

Parent JM, Vexler ZS, Gong C, Derugin N, Ferriero DM (2002) Rat forebrain neurogenesis and striatal neuron replacement after focal stroke. Ann Neurol 52:802-813.

Parmar M, Skogh C, Bjorklund A, Campbell K (2002) Regional specification of neurosphere cultures derived from subregions of the embryonic telencephalon. Mol Cell Neurosci 21:645-656.

Parmar M, Skogh C, Englund U (2003a) A transplantation study of expanded human embryonic forebrain precursors: evidence for selection of a specific progenitor population. Mol Cell Neurosci 23:531-543.

Parmar M, Sjoberg A, Bjorklund A, Kokaia Z (2003b) Phenotypic and molecular identity of cells in the adult subventricular zone. in vivo and after expansion in vitro. Mol Cell Neurosci 24:741-752.

Reynolds BA, Tetzlaff W, Weiss S (1992) A multipotent EGF-responsive striatal embryonic progenitor cell produces neurons and astrocytes. J Neurosci 12:4565-4574.

Roelink H, Porter JA, Chiang C, Tanabe Y, Chang DT, Beachy PA, Jessell TM (1995) Floor plate and motor neuron induction by different concentrations of the amino-terminal cleavage product of sonic hedgehog autoproteolysis. Cell 81:445-455.

Skogh C, Eriksson C, Kokaia M, Meijer XC, Wahlberg LU, Wictorin K, Campbell K (2001) Generation of regionally specified neurons in expanded glial cultures derived from the mouse and human lateral ganglionic eminence. Mol Cell Neurosci 17:811-820.

Skogh C, Parmar M, Campbell K (2003) The differentiation potential of precursor cells from the mouse lateral ganglionic eminence is restricted by in vitro expansion. Neuroscience 120:379-385.

Stenman J, Toresson H, Campbell K (2003) Identification of two distinct progenitor populations in the lateral ganglionic eminence: implications for striatal and olfactory bulb neurogenesis. J Neurosci 23:167-174.

Szucsik JC, Witte DP, Li H, Pixley SK, Small KM, Potter SS (1997) Altered forebrain and hindbrain development in mice mutant for the Gsh-2 homeobox gene. Dev Biol 191:230-242.
Thor S, Ericson J, Brannstrom T, Edlund T (1991) The homeodomain LIM protein Isl-1 is expressed in subsets of neurons and endocrine cells in the adult rat. Neuron 7:881-889.

Toresson H, Campbell K (2001) A role for Gsh1 in the developing striatum and olfactory bulb of Gsh2 mutant mice. Development 128:4769-4780.

Toresson H, Mata de Urquiza A, Fagerstrom C, Perlmann T, Campbell K (1999) Retinoids are produced by glia in the lateral ganglionic eminence and regulate striatal neuron differentiation. Development 126:1317-1326.

Toresson H, Potter SS, Campbell K (2000) Genetic control of dorsal-ventral identity in the telencephalon: opposing roles for Pax6 and Gsh2. Development 127:4361-4371.

Wang HF, Liu FC (2001) Developmental restriction of the LIM homeodomain transcription factor Islet-1 expression to cholinergic neurons in the rat striatum. Neuroscience 103:999-1016.

Wichterle H, Garcia-Verdugo JM, Herrera DG, Alvarez-Buylla A (1999) Young neurons from medial ganglionic eminence disperse in adult and embryonic brain. Nat Neurosci 2:461-466.

Wichterle H, Turnbull DH, Nery S, Fishell G, Alvarez-Buylla A (2001) In utero fate mapping reveals distinct migratory pathways and fates of neurons born in the mammalian basal forebrain. Development 128:3759-3771.

Winkler C, Fricker RA, Gates MA, Olsson M, Hammang JP, Carpenter MK, Bjorklund A (1998) Incorporation and glial differentiation of mouse EGF-responsive neural progenitor cells after transplantation into the embryonic rat brain. Mol Cell Neurosci 11:99-116.

Yamamoto S, Nagao M, Sugimori M, Kosako H, Nakatomi H, Yamamoto N, Takebayashi H, Nabeshima Y, Kitamura T, Weinmaster G, Nakamura K, Nakafuku M (2001) Transcription factor expression and Notchdependent regulation of neural progenitors in the adult rat spinal cord. J Neurosci 21:9814-9823.

Yun K, Potter S, Rubenstein JL (2001) Gsh2 and Pax6 play complementary roles in dorsoventral patterning of the mammalian telencephalon. Development 128:193-205.

Yun K, Garel S, Fischman S, Rubenstein JL (2003) Patterning of the lateral ganglionic eminence by the Gsh1 and Gsh2 homeobox genes regulates striatal and olfactory bulb histogenesis and the growth of axons through the basal ganglia. J Comp Neurol 461:151-165.

Zappone MV, Galli R, Catena R, Meani N, De Biasi S, Mattei E, Tiveron C, Vescovi AL, Lovell-Badge R, Ottolenghi S, Nicolis SK (2000) Sox2 regulatory sequences direct expression of a (beta)-geo transgene to telencephalic neural stem cells and precursors of the mouse embryo, revealing regionalization of gene expression in CNS stem cells. Development 127: 2367-2382. 